九州大学学術情報リポジトリ

Kyushu University Institutional Repository

\title{
Recurrent local resistance breakdown of epitaxial BaTi0_3 heterostructures
}

Watanabe, Yukio

Kyushu Institute of Technology

D. Sawamura

Kyushu Institute of Technology

Okano, Motochika

Kyushu Institute of Technology

ht tp://hdl. hand le. net/2324/4354928

出版情報: Applied physics letters. 72 (19), pp.2415-2417, 1998-03-11. American Institute of Physics

バージョン:

権利関係: (C) 1998 American Institute of Physics. 


\title{
Recurrent local resistance breakdown of epitaxial $\mathrm{BaTiO}_{3}$ heterostructures
}

\author{
Y. Watanabe, ${ }^{\text {a) }}$ D. Sawamura, and M. Okano \\ Kyushu Institute of Technology, Kitakyushu 804, Japan
}

(Received 19 December 1997; accepted for publication 6 March 1998)

\begin{abstract}
Leakage current through epitaxial $\mathrm{BaTiO}_{3}$ films was investigated to clarify the difference between the characteristics of nanometer and millimeter-size metal contacts. $\mathrm{SrTiO}_{3}: \mathrm{Nb}$ bottom electrode revealed genuine properties of a single metal $/ \mathrm{BaTiO}_{3}$ contact and demonstrated that breakdown voltage and leakage current density at both nanometer and millimeter-size contacts were controlled by the Schottky barrier. However, in marked contrast with millimeter-size contacts, nanometer-size contacts conducted little current below breakdown voltage and repeatedly exhibited abrupt breakdowns having a giant current density $>10 \mathrm{~A} \mathrm{~mm}^{-2}$. The breakdown field was as high as $0.45 \mathrm{MV} \mathrm{cm}^{-1}$ at the forward bias, while no breakdown occurred up to $0.5 \mathrm{MV} \mathrm{cm}^{-1}$ at the reverse bias. () 1998 American Institute of Physics. [S0003-6951(98)00719-0]
\end{abstract}

Leakage current through dielectric and ferroelectric films such as $(\mathrm{Ba}, \mathrm{Sr}) \mathrm{TiO}_{3}(\mathrm{BST})$ has been investigated by many researchers. ${ }^{1-6}$ To understand its origin, epitaxial ferroelectric heterostructure is advantageous. Additionally, a highly reproducible memory effect of the current through the heterostructures has been reported, ${ }^{4-6}$ which is potentially important for a high density memory combined with a scanning force microscope (SPM). ${ }^{7}$

In the studies of leakage current through ferroelectric heterostructures, electrical contacts having a large lateral dimension, typically $0.01 \mathrm{~mm}^{2}$, have been employed. However, the effects of grain boundaries and ferroelectric domain walls were supposed to be substantial. Therefore, measurement of leakage current through a single-domained portion of an epitaxial film or a high-quality single crystal is indispensable to clarify the nature of the intrinsic current conduction. However, few such approaches have been reported so far. ${ }^{7}$

SPM is widely used to measure nm-scale electrical conduction in semiconductors, nanometer-scale piezoelectric properties, and ferroelectric domain configuration. ${ }^{8-10} \mathrm{We}$ chose epitaxial $\mathrm{BTO}\left(\mathrm{BaTiO}_{3}\right)$ film on a STON $(0.5 \mathrm{wt} \% \mathrm{Nb}$ doped $\mathrm{SrTiO}_{3}$ ) substrate, because the BTO/STON junction is regarded approximately as ohmic and possesses a clean interface due to a good lattice matching [Fig. 1(a)]. Therefore, in a metal BTO/STON heterostructure, the current is controlled mostly by the metal/BTO interface, and its characteristics are expected to reveal genuine properties of the metal/ BTO contact. The present SPM study uncovers currentvoltage $(I-V)$ characteristics of BTO film having a nanometer-size electrical contact. Here, the contact was placed at a central part of a ferroelectric domain and was much smaller than the typical sizes of domains.

BTO films were epitaxially grown by pulse laser deposition on STON that served as an $n$-type bottom electrode. The x-ray $\theta / 2 \theta$ scan and the x-ray pole figure diffractometry verified three-dimensional alignment of the $\mathrm{BTO}(001)$ with the $\operatorname{STON}(001) .{ }^{11}$ No secondary phase was detected by the depth profile using secondary ion mass spectroscopy and $\mathrm{x}$ -

${ }^{a)}$ Electronic mail: ynabe@tobata.isc.kyutech.ac.jp ray diffractometry. The BTO films were $\sim 100 \mathrm{~nm}$ thick and tetragonal in crystal symmetry, suggesting a ferroelectric phase at room temperature (RT). The remanent polarization measured by a Sawyer-Tower circuit was $1-2 \mu \mathrm{C} / \mathrm{cm}^{2}$. Both millimeter and nanometer-size contacts were made on the BTO surface as shown in Fig. 1(b). Metal films of Pt, Au, and $\mathrm{Al}$ with surface area of $0.01-1 \mathrm{~mm}^{2}$ were deposited as millimeter-size top electrodes. A commercial SPM, i.e., an atomic force microscope (AFM), was used to make a nanometer-size contact on BTO surface. The top electrode, i.e., an AFM tip (hereafter tip), was made of $\mathrm{Si}_{3} \mathrm{~N}_{4}$ coated with 50-100 nm thick Pt film. The resonant frequency of the cantilever was $10-20 \mathrm{kHz}$, and the tip contacted the BTO surface with a repulsive force of $10-30 \mathrm{nN}$. One $I-V$ curve was measured approximately in $3.5 \mathrm{~min}$, and polarity of the bias voltage was defined at the top electrode. All measurements were performed in a dry atmosphere at RT. A BTO of a modest resistance was used below, but the results for millimeter-scale contacts were confirmed to be qualitatively similar to those of high resistance BTOs. [No current was observed in the high resistance BTO films (200 $\mathrm{nm}$ thick) for applied voltage of $11 \mathrm{~V}$.]

Figure 2 shows $I-V$ characteristics at a Pt electrode hav-

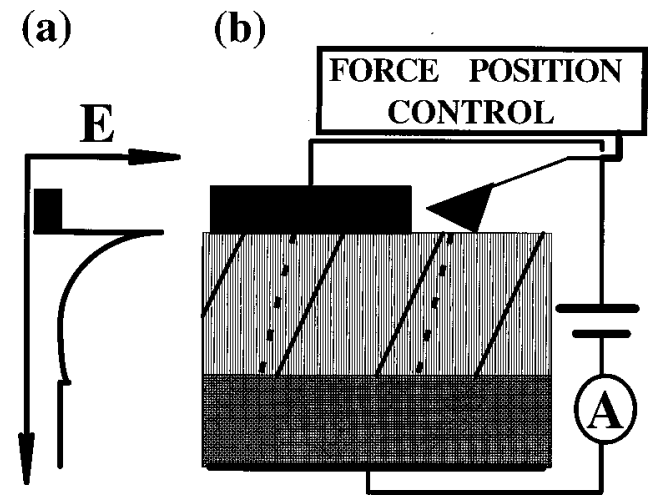

FIG. 1. Diagram showing the bottom of the conduction bands of BTO and STON and the Fermi level of Pt at zero bias voltage (a). Experimental setup for $I-V$ measurements using an AFM with a Pt-coated tip and metal electrodes on BTO surface (b). The tilted solid dotted lines show domain and grain boundaries, respectively. 

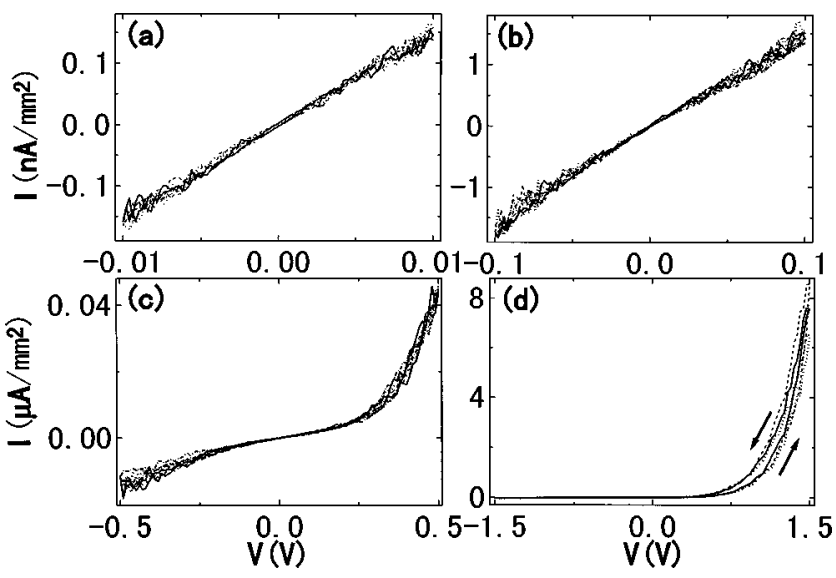

FIG. 2. $I-V$ curves of $\mathrm{Pt}$ (area: $0.4 \mathrm{~mm}^{2}$ )/BTO/STON at RT. The dotted, dashed, solid, and dashed-dotted lines correspond to the first, second, third, and fourth scan. (a) $\pm 0.01 \mathrm{~V}$, (b) $\pm 0.1 \mathrm{~V}$, (c) $\pm 0.5 \mathrm{~V}$, (d) $\pm 1.5 \mathrm{~V}$ scan. The arrows show the direction of the change.

ing surface area of $0.4 \mathrm{~mm}^{2}$. In each of Figs. 2(a) and 2(d), the three lines correspond to three consecutive $I-V$ measurements. In each of Figs. 2(b) and 2(c), the four lines correspond to three consecutive measurements and one after the measurements for Fig. 2(d). Figures 2(b) and 2(c) demonstrate that no conduction properties were changed after the large current flow [Fig. 2(d)].

$I-V$ characteristics were ohmic at low voltages [Figs. 2(a) and 2(b)] and Schottky diodelike at high voltages [Figs. 2(c) and 2(d)]. Reproducible hystereses at the forward bias were due to conductivity enhancement, while those at the reverse bias were due to relaxation. Overall $I-V$ characteristics were essentially the same at $\mathrm{Pt}, \mathrm{Au}$, and $\mathrm{Al}$ electrodes, although $\mathrm{Al}$ electrodes were the most conductive, and $\mathrm{Pt}$ electrodes were the most resistive. The temperature dependence of the current density was activation-type. These results indicate that the conduction through metal/BTO/STON at high bias voltage was limited by the metal/BTO interface having a Schottky-type potential barrier. The polarity of the forward bias, the hysteresis, and the voltage dependence are consistent with the previous reports on $\mathrm{Pb}$-based ferroelectric heterostructures. ${ }^{4-6}$

Before performing local $I-V$ measurements, ferroelectric domain configurations of the BTO film used for the results in Fig. 2 were investigated by imaging electric force distribution on the surface in a noncontact mode. ${ }^{10}$ Topographic images were then obtained in a contact mode to identify grain boundaries. Only in electric force images of areas without metal films, we observed $20-\mu \mathrm{m}$-wide parallel stripes accompanied by a few micron wide stripes. No such patterns were visible in topographic images. Therefore, these stripes were considered as $90^{\circ}$ ferroelectric domains. No clear structure corresponding to micron-size grains were detected, which was consistent with the absence of clear grain structure in the high-resolution SEM cross section images.

Subsequently, $I-V$ characteristics of specific points were measured. When a tip was on a metal electrode, $I-V$ characteristics similar to Fig. 2 were observed, indicating that the tip force was sufficient to make an electrical contact. Figure 3 shows typical local $I-V$ characteristics measured successively with a tip placed directly on the BTO surface at

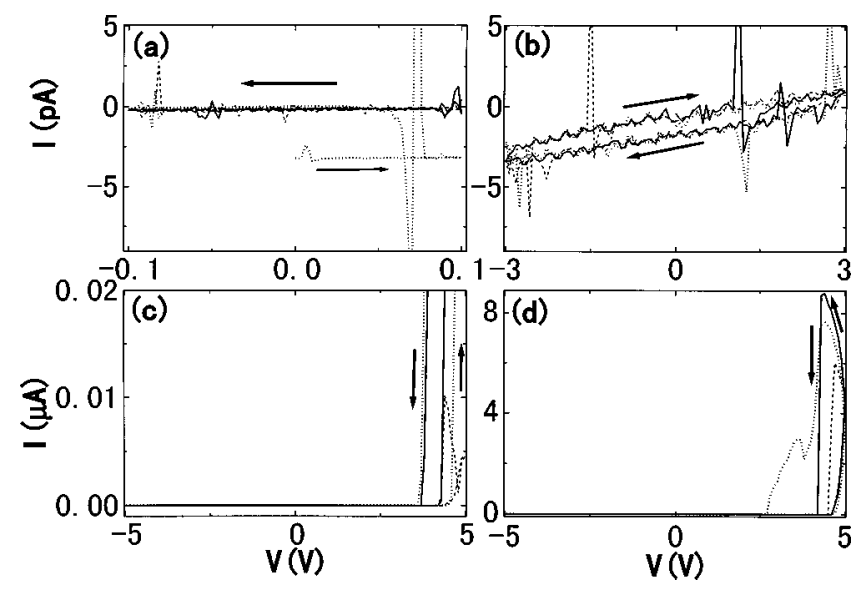

FIG. 3. $I-V$ curves of Pt coated tip/BTO/STON at RT. (a) $\pm 0.1 \mathrm{~V}$, (b) \pm 3 , (c), (d) $\pm 5 \mathrm{~V}$ scan. Each panel shows $I-V$ curves of three consecutive voltage scans, and the arrows show the direction of the change. The meaning different lines are same as Fig. 2.

a central part of a 20- $\mu$ m-wide domain. Each panel in Fig. 3 displays $I-V$ hystereses of three consecutive measurements. $I-V$ characteristics were almost independent of a small increase of the tip contact force. Additionally, overall $I-V$ characteristics at different locations were similar, and the details of their dependence on location will be reported elsewhere.

Little current was observed until the bias voltage first exceeded $+4.5 \mathrm{~V}$. The current shown in Fig. $3(\mathrm{~b})$ and the current at negative bias shown in Fig. 4(b) were not true currents through the BTO film, because a similar current density was measured when the tip was detached from the BTO surface, or Pt film was peeled off from the tip. Except for the pair of large spikes in Fig. 3(a), the spikes in Figs. $3(\mathrm{a})$ and 3(b) were probably due to instrumental noise. In Fig. 3(a), a bias current is indicated by the dotted lines. Bias current was consistently observed just after the tip contacted on the BTO surface and was likely related with the piezoelectricity or surface charges shielding the spontaneous polarization. $^{12}$

According to Fig. 4(a) (millimeter-size contact), we estimate the current intensity of $\sim 0.1 \mathrm{nA}$ at $3 \mathrm{~V}$ and $1 \mathrm{nA}$ at 4 $\mathrm{V}$ by assuming a tip/BTO contact area of $10^{4} \mathrm{~nm}^{2}$. The observed current intensity in Fig. 4(b) (nanometer-size contact)
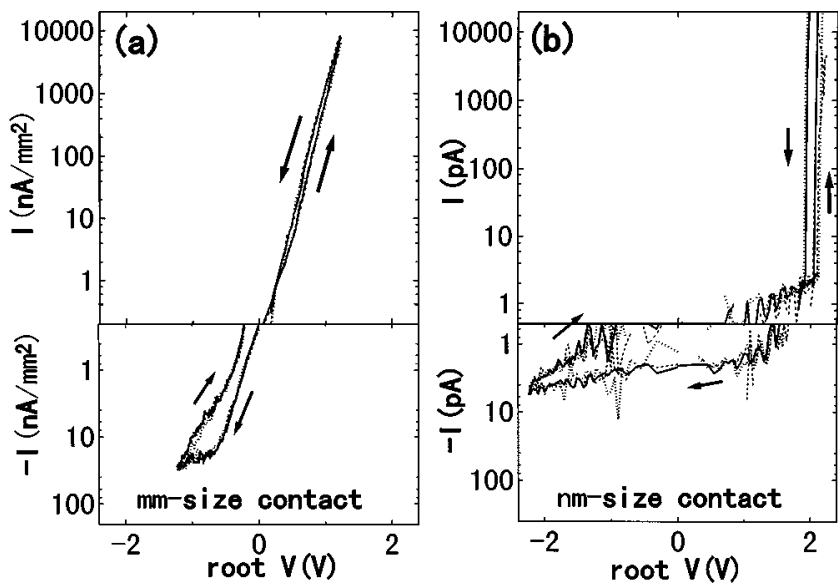

FIG. 4. Replot of Fig. 2(d) [(a)] and Fig. 3(c) [(b)]. Arrows show the direction of the change. The mean different lines are the same as Fig. 2. 
was more than three orders of magnitude lower than the above values. The discrepancy enlarges if we assume a larger contact area. This implies that the resistance at the millimeter-size electrodes was limited by conductive paths such as domain boundaries, grain boundaries, dislocations, or defect aggregates. Namely, BTO film is intrinsically a good insulator, at least, up to $0.4 \mathrm{MV} \mathrm{cm}^{-1}$.

In Figs. 3(c) and 3(d), abrupt current intensity jumps which occurred repeatedly are shown. The dotted lines in Fig. 3(c) show the $I-V$ curve for the first $5 \mathrm{~V}$ scan, and the replot of Fig. 3(c), i.e., Fig. 4(b), clearly shows a threshold at 4.6 V. The threshold of the jump was well defined and changed only slightly with repeated measurements. On the other hand, the resistive state reappeared at various voltages between 2.7 and $4.3 \mathrm{~V}$ from measurement to measurement.

In Fig. 4(b), the conductivity is shown to jump by more than six orders of magnitude, which corresponds to the conductivity enhancement seen in Fig. 4(a). The maximum current density is $\sim 1 \mathrm{kA} / \mathrm{mm}^{2}$ if we assume a tip contact area of $10^{4} \mathrm{~nm}^{2}$ and a direct current conduction through the BTO film beneath the contact. Despite the huge current intensity, the insulating property was still preserved below $4 \mathrm{~V}$ thereafter. Furthermore, except for the disappearance of the bias current, no change was found in the $I-V$ curves of $\pm 0.1 \mathrm{~V}$ scans after the current jumps.

The exceptionally abrupt increase of the current shown in Figs. 3(c) and 3(d) and the subsequent current increase during the voltage decrease from 5 to $4.5 \mathrm{~V}$ were inexplicable by the conduction limited by the Schottky barrier. One explanation is surface conduction to the vicinity of a leakage path. This surface conduction was not due to a trivial cause such as water adsorption, because no conduction was observed below $+4 \mathrm{~V}$. The tip was located $10 \mu \mathrm{m}$ away from domain boundaries and $30 \mu \mathrm{m}$ away from the nearest metal electrode. The leakage current was nearly independent of the distance between the tip and the electrode (or the domain boundary), supporting the absence of a water-induced surface conduction.

An alternative viewpoint is to regard the current jump as an insulator breakdown, for which various mechanisms, e.g., the impact ionization, are proposed. However, the present breakdown was recoverable unlike the intensively investigated breakdown of $\mathrm{SiO}_{2} \cdot{ }^{13}$ One possible explanation may be local evaporation of $\mathrm{Pt}$ at the contact by Joule heating, often called as a self-healing effect. ${ }^{14}$ Additionally, the breakdown occurred only in the forward bias condition of the Pt/BTO Schottky barrier, suggesting that injected electrons from the barrier initiated the breakdown.

Below $0.45 \mathrm{MV} \mathrm{cm}^{-1}$ at the forward bias, leakage current was well below the measurement limit, although BTO films are usually considered to be much leakier than BST and $(\mathrm{Pb}, \mathrm{La})(\mathrm{Zr}, \mathrm{Ti}) \mathrm{O}_{3}$ films. At the reverse bias, no leakage current was detected even at $0.5 \mathrm{MV} \mathrm{cm}^{-1}$. This indicates that the BTO film is intrinsically more insulating than conventionally thought and that the increase of leakage current with decreasing thickness is explicable by a percolation mechanism. This consideration also suggests a possibility of a superior insulating property of $\mathrm{Pt} / \mathrm{BTO} / \mathrm{Pt}$, at least, up to $\pm 0.5 \mathrm{MV} \mathrm{cm}^{-1}$, because one of the Pt/BTO contacts is always reverse biased.

In conclusion, nanometer-scale electrodes exhibited $I-V$ characteristics strikingly different from the Schottky diodelike characteristics of millimeter-size electrodes: a very high resistivity and an abrupt giant breakdown. Nonetheless, the breakdown voltage was controlled by the Schottky barrier at both sizes of contacts.

This work is performed through the support of Grant-inAid for Scientific Research from the Ministry of Education, Science and Sports. The authors acknowledge the Corning Grant Award'96 and proofreading by Professor S. Takagi.

${ }^{1}$ P. C. Joshi and S. B. Krupanidhi, J. Appl. Phys. 73, 7627 (1993); G. R. Fox and S. B. Krupanidhi, ibid. 74, 1949 (1993); C. Sudama, A. Campbell, P. Maniar, R. Jones, R. Moazzami, C. Mogab, and J. Lee, ibid. 75, 1014 (1994).

${ }^{2}$ G. W. Dietz, W. Antpohler, M. Klee, and R. Waser, J. Appl. Phys. 78, 6113 (1995).

${ }^{3}$ S. R. Gilbert, L. A. Willis, B. W. Wessels, J. L. Schindler, J. A. Thomas, and C. R. Kannewurf, J. Appl. Phys. 80, 969 (1996).

${ }^{4}$ Y. Watanabe, Y. Matsumoto, M. Tanamura, H. Asami, and A. Kato, Physica C 235-240, 739 (1994); Y. Watanabe, Appl. Phys. Lett. 66, 28 (1995).

${ }^{5}$ P. Blom, R. Wolf, J. Cillessen, and M. Krijn, Phys. Rev. Lett. 73, 2107 (1994).

${ }^{6}$ K. Gotoh, H. Tamura, H. Takeuchi, and A. Yoshida, Jpn. J. Appl. Phys., Part 1 35, 39 (1996).

${ }^{7}$ C. Yoshida, H. Tamura, A. Yoshida, and N. Yokoyama, Extended Abstract 57th Autumn Meeting Japan Society Appl. Phys. 465 (1996); Y. Watanabe, Jpn. Patent Appl. Disclosure No. 7-263646 (1995).

${ }^{8}$ S. Hosaka, S. Hosoki, K. Tanaka, K. Horiguchi, and N. Natsuaki, Appl. Phys. Lett. 53, 487 (1988); O. Votel and M. Tanimoto, J. Appl. Phys. 77, 2358 (1995).

${ }^{9}$ F. Saurenbach and B. D. Terris, Appl. Phys. Lett. 56, 1703 (1990); P. Güthner and Dransfeld, ibid. 61, 1137 (1992); K. Franke, J. Besold, W. Haessler, and C. Seegebarth, Surf. Sci. Lett. 302, L283 (1994).

${ }^{10}$ R. Lüthi, H. Haefke, K.-P. Meyer, E. Meyer, L. Howald, and H.-J. Güntherodt, J. Appl. Phys. 74, 7461 (1993).

${ }^{11}$ Y. Watanabe, Y. Matsumoto, H. Kunitomo, M. Tanamura, and E. Nishimoto, Jpn. J. Appl. Phys., Part 1 33, 5182 (1994).

${ }^{12}$ Y. Watanabe, Phys. Rev. B 57, 789 (1998).

${ }^{13}$ J. J. O'Dwyer, The Theory of Electrical Conduction and Breakdown in Solid Dielectrics (Clarendon, Oxford, 1973); I. Chen, S. E. Holland, and C. Hu, IEEE Trans. Electron Devices ED-32, 413 (1985).

${ }^{14}$ This model needs to be modified to explain the subsequent recurrent current jumps in Fig. 4. 